http://dx.doi.org/10.15762/ZH.2017.38

\author{
RAFAŁ KUBICKI \\ (Uniwersytet Gdański)
}

\title{
WYKAZ CZYNSZÓW I SŁUŻB KOMTURSTWA CZŁUCHOWSKIEGO \\ Z 1446 ROKU WRAZ Z INWENTARZEM KONI, UZBROJENIA I ZAPASÓW KUCHNI NA ZAMKU W CZŁUCHOWIE
}

Słowa kluczowe: zakon krzyżacki, gospodarka wiejska, Pomorze Wschodnie

Podejmując próbę rekonstrukcji stanu gospodarki wiejskiej we władztwie zakonu krzyżackiego w Prusach, mamy do dyspozycji szereg źródeł o charakterze przekrojowym. Do najważniejszych wśród nich należą wykazy czynszów pobieranych przez Zakon z wsi i obiektów gospodarczych (m.in. młynów i karczem). Dają one możliwość w miarę całościowej prezentacji stanu gospodarki wiejskiej (liczby łanów zagospodarowanych i pustych) oraz infrastruktury gospodarczej w chwili sporządzenia wykazu. Zestawienie kilku przekazów tego typu, dotyczących tego samego terenu, pozwala zaś na śledzenie przemian następujących w sieci osadniczej i ogólnym stanie zagospodarowania. Opisana sytuacja dotyczy m.in. terenu komturstwa człuchowskiego. Dotychczas opublikowano dwa źródła o charakterze przekrojowym, powstałe w pierwszej połowie XV w., które uwzględniają to komturstwo. Mowa tu o wykazie krzywd i strat archidiecezji gnieźnieńskiej z 1436 r. $^{1}$ i zestawieniu czynszów komturstwa człuchowskiego z 1437 r., ogłoszonym w ramach tzw. wielkiej księgi czynszowej zakonu krzyżackiego, zawierającej w rzeczywistości dane pozyskane w ramach wizytacji krajowej ${ }^{2}$. Dodajmy, że ważne informacje, dopełniające oba przekazy, przynosi wykaz dziesięcin sporządzony w ramach również opublikowanej wizytacji dóbr arcybiskupstwa gnieźnieńskie-

${ }^{1}$ Marian Biskup, Wykaz krzywd i strat wyrządzonych archidiecezji gnieźnieńskiej w okręgach człuchowskim i tucholskim z roku 1436, Zapiski Historyczne (dalej cyt. ZH), t. 69: 2004, Z. 1, s. 111-123.

${ }^{2}$ Das grosse Zinsbuch des Deutschen Ritterordens (1414-1438), hrsg. v. Peter G. Thielen, Marburg 1958, s. 108-113. 
go z 1512 r. ${ }^{3}$ W tej sytuacji każdy kolejny przekaz tego rodzaju stanowi bardzo ważny materiał porównawczy, pomocny w dalszych badaniach nad osadnictwem i gospodarką wiejską.

W związku z tym publikujemy wykaz czynszów i służb komturstwa człuchowskiego z 1446 r., który w zasadniczej swej części ma podobny charakter do wspomnianego zestawienia czynszów komturstwa z 1437 r., zawiera jednak zazwyczaj bardziej szczegółowe dane na ten temat ${ }^{4}$. W pierwszej części wykazu umieszczono zestawienie koni, dane na temat zaopatrzenia zbrojowni i zapasów zgromadzonych w kuchni na zamku w Człuchowie ${ }^{5}$. Dalej znajduje się, stanowiący zasadniczą część zestawienia, opis stanu poszczególnych osad wiejskich. Obok ogólnej liczby łanów czynszowych odnotowuje on nie tylko liczbę łanów faktycznie zasiedlonych, z wysokością płaconego z nich czynszu, lecz także liczbę łanów pustych i tych znajdujących się w trakcie ponownego zagospodarowywania (w okresie wolnizny), z podaniem terminu wpłaty pierwszego czynszu. W wykazie uwzględniono także czynsze pobierane $\mathrm{z}$ działających na terenie komturstwa karczem i młynów, zamienione na czynsz w pieniądzu dawne świadczenia dóbr rycerskich na prawie polskim (ekwiwalent w pieniądzu za krowę i świnię - opłata określona jako swen und ku tzinß), oraz tzw. owies leśny (walthaber) - opłatę za pozwolenie zbierania chrustu i wypasu bydła w lasach bukowych Zakonu. W ostatniej części omawiane źródło zawiera zestawienie służb rycerskich, w którym wymieniono zarówno dobra rycerskie obsadzone chłopami i służące zbrojnie, jak i te opuszczone, wówczas niezdolne do wypełniania swych zobowiązań militarnych ${ }^{6}$.

Prezentowany tu przekaz przechowywany jest obecnie w Geheimes Staatsarchiv Preußischer Kulturbesitz (Tajne Archiwum Pruskich Dóbr Kultury) w Berlinie Dahlem (GStA PK), w obrębie zbiorów dawnego archiwum zakonu krzyżackiego. Umieszczono go wśród listów (Ordensbriefarchiv), pod numerem 28953 (sygnatura dawna Convents-Rechnungen f.). W drukowanym

${ }^{3}$ Visitationes bonorum archiepiscopatus necnon capituli Gnesnensis saeculi XVI, ed. Bolesław Ulanowski, Kraków 1920, s. 334-336.

${ }^{4}$ Źródło to znane jest już badaczom. Wykorzystywali je w swych pracach: Klemens BrUSKI, Lokalne elity rycerstwa na Pomorzu Gdańskim w okresie panowania zakonu krzyżackiego studium prozopograficzne, Gdańsk 2002; Rafał KuвICKI, Młynarstwo w państwie zakonu krzyżackiego $w$ Prusach $w$ XIII-XV wieku (do 1454 r.), Gdańsk 2012; Michał TArgowski, Na prawie polskim i niemieckim. Kształtowanie się ziemskiej własności szlacheckiej na Pomorzu Gdańskim w XIII-XVI wieku, Warszawa 2014. Niestety nie uwzględnił tego przekazu Maksymilian Grzegorz zob. idem, Słownik historyczno-geograficzny ziemi chojnickiej w granicach komturstwa człuchowskiego, Chojnice 2005 (dalej cyt. SHGZCH).

${ }^{5}$ Fragment ten został opublikowany w Das grosse Ämterbuch des Deutschen Ordens, hrsg. v. Walther Ziesemer, Danzig 1921 (dalej cyt. GA), s. 670-671.

${ }^{6}$ M. TARgowski, op.cit., s. 138, przyp. 113. 
regestariuszu korespondencji Zakonu opisano go jako: Inwentarz koni i broni, uzbrojenia i kuchni na zamku w Człuchowie; wykaz łanów czynszowych i służb w okręgu człuchowskim („Inventar der Pferde und Waffen, des Geschützes und der Küche auf dem Schlosse Schlochau; Verzeichniß der Zinshufen und Dienste des Gebietes Schlochau") ${ }^{7}$. Wykaz spisano na kartach papierowych złożonych w format dudki o wymiarach około $10 \times 27 \mathrm{~cm}$. Tekst spisano w języku niemieckim, minuskułą gotycką, pismem czytelnym z użyciem nielicznych abrewiacji. Kolejne karty mają foliację od 1 do $11 \mathrm{v}$.

W przygotowaniu tekstu do druku korzystano częściowo z instrukcji wydawniczej Adama Wolffa ${ }^{8}$. W celu ukazania podstawy źródłowej użyto następujących symboli z instrukcji: /- -/ skreślenie, [s] potwierdzenie błędu lub indywidualizacji zapisu w podstawie wydania, (?) wątpliwość. Abrewiacje rozwiązano bez zaznaczania tego $\mathrm{w}$ tekście. W nawiasach kwadratowych uzupełniono daty roczne wpłaty czynszu. Występujace $\mathrm{w}$ tekście oryginalnym cyfry rzymskie zastąpiono arabskimi bez oznaczania tego w przypisach. Uproszczono również zapis kwot np: „441 mr” zamiast „400 und 41 mr”. W edycji ujednolicono zapis jednostek obrachunkowych i miary objętości, stosując następujące skróty: $\mathrm{d}$ - denarius (fenig, denar), $\mathrm{f}$ - firdung (wiardunek), $\mathrm{mr}$ - mark (grzywna), sc - scot (skojec), sch - scheffel (korzec), lb - libra (funt). Obowiązujące ówcześnie w państwie krzyżackim miary zbożowe to: 1 korzec (55,0 litrów) $=4$ wiertle $=16$ miarek, zaś system obrachunkowy: 1 grzywna $=4$ wiardunki $=24$ skojce $=60$ szelągów $=720$ fenigów $($ denarów). Oszczędność miejsca $\mathrm{w}$ druku zadecydowała o nieoddawaniu oryginalnego układu tekstu, lecz zastąpieniu go własnym, wprowadzonym przez wydawcę, uporządkowaniem graficznym.

${ }^{7}$ Regesta historico-diplomatica Ordinis S. Mariae Theutonicorum 1198-1525 (dalej cyt. RHDO), Pars I, vol. 3: (1511-1525), bearb. v. Erich JoAchim, hrsg. v. Walther Hubatsch, nr 28953, s. 534.

${ }^{8}$ Adam WolfF, Projekt instrukcji wydawniczej dla pisanych źródeł historycznych do połowy XVI wieku, Studia Źródłoznawcze, t. 1: 1957, s. 155-184. 


\section{ANEKs ŹRÓDŁOWY}

Wykaz czynszów i służb komturstwa człuchowskiego z 1446 r. wraz z inwentarzem koni, uzbrojenia i zapasów kuchni na zamku w Człuchowie

Org.: GStA PK, XX. HA, Ordensbriefarchiv nr 28953.

Uw.: Sygnatura dawna (Convents-Rechnungen f.).

Regest: RHDO, Pars 1, vol. 3 (1511-1525), nr 28953, s. 534.

\section{[k. $1 r]$ Schlochow}

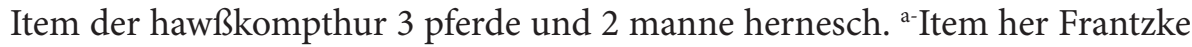
Bryger $^{1}$. Item her Johannes Hoensteyn ${ }^{2 \text {-a }}$. Item der waltmeister 3 pferde 2 manne hernesch. Item Heppenberg 33 pferde 1 manne harnesch. Item Dynyes Tzarte $^{4} 2$ pferde 1 manne harnesch. Item Techwitz 3 pferde 1 manne harnesch. Item pferdemarschalk 3 pferde und zcu einem manne harnesch. Item 3 pferde nicht harnesch. Item der kellermeister 2 pferde. Item der thormeyster 1 pferd. Item der pfleger von der Baldeburg ${ }^{6}$ hoth 3 pferde und 1 manne harnesch. Item Hose $^{7} 3$ pferde. Item der kuchmeister zcu 1 manne harnesch. Item Johan Rost $^{8}$ nicht pferde nicht harnesch.

Geschoß

Item 9 steynbuchsen. Item 8 kamern zcu buchsen. Item 19 lotbuchsen. Item $51 / 2$ tonen pulver. Item 36 armbrost in der dreßkammer. Item 1 schok minus 4 orter armbrost in der puluerkamer. Item 200 schok pfeyle und 40 laden mit pfeyle uffim thorme. Item 10 schok und hundert schok pfeyle in der puluerkamer. Item harnesch zcu 5 mannen.

a--a Po prawej stronie dopisek, odnoszacy się do obu osób: prysterbrüder.

${ }^{1}$ Frantzke Bryger kapłan, bliżej nieznany.

${ }^{2}$ Johanes Hoenstein kapłan wzmiankowany w 1437 r. w konwencie w Toruniu, GA, s. 449.

${ }^{3}$ Heppenberg, może tożsamy z wzmiankowanym w 1451 r. Conradem Heppenberg z konwentu w Brandenburgu, GA, s. 240.

${ }^{4}$ Dynyes Tzarte. W 1454 r. wzmiankowany był w składzie konwentu Antonius Czart, RHDO, Pars I, vol 1 (1198-1454), bearb. v. E. Joachim, hg. v. W. Hubatsch (Göttingen: Vandenhoeck \& Ruprecht, 1948), nr 12862, s. 836.

${ }^{5}$ Heynrich Techwitz wzmiankowany w $1413 \mathrm{r}$. w konwencie w Człuchowie jako rycerz Zakonu pochodzący z Miśni, zob. Marian BIsKup, Skład konwentu zakonu krzyżackiego w Człuchowie z 1413 r., ZH, t. 63: 1998, z.1, s. 127.

${ }^{6}$ Prokurator w Białym Borze.

${ }^{7}$ Hose, bliżej nieznany.

${ }^{8}$ Johann Rost, wzmiankowany również w wykazie konwentu z 1437 i 1443 r., Das grosse Zinsbuch, s. 111; GA, s. 670. 
[k. 1v] Dye kuche

Item 8 schok vlicken speckfleysch. Item 200 spyß trewge fleysch. Item 15 stukke rotwilt im saltze. Item 9 tonen saltz.

[k. 2r] Dese nochgeschrebin schryft weyset awß egentlichen alle tzinse an gelde und an getreyde awß steten und dorffern, an molen und wercherley sye sint und wye fil besatzter und unbesattzer und freyer huben im Schloweschen gebyete

Rychenaw $^{9}$ hoth 66 tzinßhuben der ist 30 besatzt und 26 zcu tzinse komen und tzinset von der hube 1/2 mr, 2 huner. Summa $13 \mathrm{mr}$. Summa: 36 huben sint wuste, 30 besatzt, item 4 huben freyheyt. Item 4 huben sullen tzinsen /-5-/ im [14]47 $7^{\text {ten }}$ jore.

Polnitz ${ }^{10}$ hoth 38 tzynßhuben der ist 29 besatzt und $23 \mathrm{zcu}$ tzinse komen und tzinset von der hube $10 \mathrm{sc}, 2$ huner. Item tzwene kretzem tzinset itzlicher 15 sc., der eine ist wuste. Summa 10 und und [s] 5 sc. Summa: 29 besatzen huben, 23 tzinsen, 6 haben freyheyt, 7 wuste. Item dy freyen sullen tzinsen im $[14] 48^{\text {ten }}$ jore.

Mawskendorff ${ }^{11}$ hoth 56 tzynßgarten der 44 besatzt und 40 zcu tzinse komen und tzinset vom garten $10 \mathrm{sc}$ minus $6 \mathrm{~d}$, 2 huner. Item ein krug der sulde tzinsen $21 / 2 \mathrm{mr}$ und tzinset $2 \mathrm{mr}$. Summa $18 \mathrm{mr}$ und 8 sc. Summa: 44 besatzt, 40 tzinsen, 4 freyen, 10 sint wuste. Item 4 freyen sullen tzinsen im [14]48 $8^{\text {ten }}$ jore.

Wenige Conitz ${ }^{12}$ hoth 41 tzinßhuben der 35 besatzt und 15 zcu tzinse komen und tzinset von der hube 14 sc, 2 huner. Item tzwene kretzem tzinsen zcu gutten $\mathrm{mr}$, der eine ist wuste. Summa /-18 mr und $8 \mathrm{sc}-/ 9 \mathrm{mr}$ und $1 \mathrm{f}$. Summa: 35 besatzt, 15 tzinsen, 20 frey, 6 wuste. Item 12 huben sullen tzinsen im

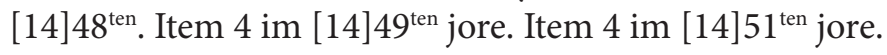

Summa $32 \mathrm{mr}$ und $1 \mathrm{f}$.

[k. 2v] Newkirche ${ }^{13}$ hoth ${ }^{\mathrm{b}-50^{-\mathrm{b}}}$ tzinßhuben der ist 36 besatzt und $14 \mathrm{zcu}$ tzinse komen und tzinset von der hube $1 / 2 \mathrm{mr}, 2$ huner. Summa $7 \mathrm{mr}$. Item tzwene kretzem sulden tzinsen itzlicher $3 \mathrm{f}$, der eine ist wuste und sal tzinsen im [14] $48^{\text {ten }}$ jore. Item: 36 besatz, 14 tzinsen, 22 freye, 14 wuste. Item 16 freyen sullen tzinsen im [14]48 ${ }^{\text {ten }}$ jore. Item 6 im [14]50 ${ }^{\text {ten }}$ jore.

\footnotetext{
b- -b Tekst nadpisany.

${ }^{9}$ Rychnowy, $5 \mathrm{~km}$ na NE od Człuchowa, SHGZCH, s. 102-103.

${ }^{10}$ Polnica, $10 \mathrm{~km}$ na N od Człuchowa, SHGZCH, s. 94-95.

${ }^{11}$ Charzykowy, $5 \mathrm{~km}$ na NW od Chojnic, SHGZCH, s. 30-31.

${ }^{12}$ Chojniczki, $5 \mathrm{~km}$ na $\mathrm{N}$ od Chojnic, SHGZCH, s. 36-37.

${ }^{13}$ Nowa Cerkiew, 7 km na E od Chojnic, SHGZCH, s. 84-85.
} 
Brysen ${ }^{14}$ hoth 47 tzinßhuben der 21 1/2 besatzt und 14 zcu tzinse komen und tzinset von der hube $14 \mathrm{sc}, 2$ huner. Summa $8 \mathrm{mr}$ und $4 \mathrm{sc}$. Item 1 kretzem tzinset $1 / 2 \mathrm{mr}$, ist wuste. Item: $61 / 2$ freyen, $251 / 2$ sint wuste. Item $31 / 2$ huben sul-

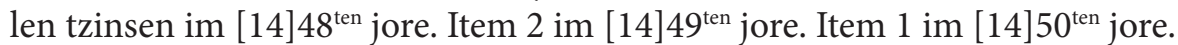

Grose Gentznik ${ }^{15}$ hoth 57 tzinßhuben der ist 19 besatzt und 15 zcu tzinse komen und tzinset von der hube 17 sc, 2 huner. Summa $10 \mathrm{mr}$ und 16 sc. Item 2 kretzem itzlicher $1 \mathrm{mr}$, sint wuste. Item 4 sint noch frey. Item 38 wuste. Item 4 freyen sullen tzinsen im [14]48 ${ }^{\text {ten }}$ jore.

Lychtenhagen ${ }^{16}$ hoth 50 tzinßhuben der ist 21 besatzt und $17 \mathrm{zcu}$ tzinse komen und tzinset von der hube $1 / 2 \mathrm{mr}, 2$ huner. Summa $81 / 2 \mathrm{mr}$. Item 1 kretzem $1 \mathrm{mr}$, ist wuste. Item 17 tzinsen. Item 4 sint noch frey. Item 29 sint wuste. Item

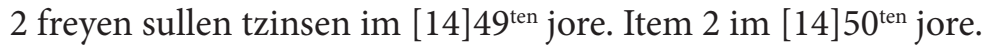

Summa $331 / 2 \mathrm{mr}$.

[k. 3r] Dampnitz ${ }^{17}$ hoth 50 tzinsshuben der ist 29 besatzt und $27 \mathrm{zcu}$ tzinse komen und tzinset von der hube $1 / 2 \mathrm{mr}$ und $1 \mathrm{sc}, 2$ huner. Item 2 kruge zcu $3 \mathrm{f}$, einer ist wuste. Summa $14 \mathrm{mr} 17$ sc minus $6 \mathrm{~d}$. Summa: 29 besatzt, 2 frey, item 19 wuste. Item 2 freyen tzinsen im [14]48 ${ }^{\text {ten }}$ jore.

Virchow ${ }^{18}$ hot 54 tzinßhuben der ist 25 besatzt und $10 \mathrm{zcu}$ tzinse komen und tzinset von der hube $1 / 2 \mathrm{mr}, 2$ huner. Summa $5 \mathrm{mr}$. Summa: 25 besatzt, item 15 freye, item 29 sint wuste. Item 4 sullen tzinsen im [14] $48^{\text {ten }}$ jore. Item $4 \mathrm{im}$ $[14] 49^{\text {ten }}$ jore. Item $7 \mathrm{im}[14] 52^{\text {ten }}$ jore.

Steynborn ${ }^{19}$ hoth 57 tzinßhuben der ist 56 besatzt und 35 zcu tzinse komen und tzinset von der hube $16 \mathrm{sc}, 2$ huner. Item 2 kretzem sullen tzinsen $3 \mathrm{mr}$ minus $1 \mathrm{f}$, der eine tzinset $5 \mathrm{f}$, der ander ist wuste. Summa $24 \mathrm{mr}$ und $14 \mathrm{sc}$. Summa: 35 tzinsen, 21 sint frey, 1 ist wuste. Item 6 sullen tzinsen im [14] $48^{\text {ten }}$.

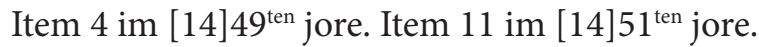

Cristfeld ${ }^{20}$ hoth /-10-/ 56 tzinßhuben der ist 22 besatzt und 19 zcu tzinse komen und tzinset von der hube $1 \mathrm{f}$. Item tzwene kretzem, einer ist wuste, der ander tzinset ${ }^{\mathrm{c}-5} \mathrm{f}^{-\mathrm{c}}$ Summa $6 \mathrm{mr}$. Summa: 22 besatzt, 19 tzinsen, 3 /-wuste-/ freye, 33 sint wuste. Item 3 freye sullen tzinsen im [14]51 $1^{\text {ten }}$ jore.

Summa 49 mr.

${ }^{c--c}$ Tekst nadpisany ze znakiem wstawienia.

${ }^{14}$ Brzeźno Człuchowskie, 8 km na E od Człuchowa, SHGZCH, s. 27-28.

${ }^{15}$ Jęczniki Wielkie, $6 \mathrm{~km}$ na SE od Człuchowa, SHGZCH, s. 63-64.

${ }^{16}$ Ględowo, 3 km na SW od Człuchowa, SHGZCH, s. 56-57.

${ }^{17}$ Dębnica, $3 \mathrm{~km}$ na S od Człuchowa, SHGZCH, s. 51-53.

${ }^{18}$ Wierzchowo Człuchowskie, $8 \mathrm{~km}$ na SE od Człuchowo, SHGZCH, s. 117.

${ }^{19}$ Słupia, 6 km na NE od Debrzna, SHGZCH, s. 107-108.

${ }^{20}$ Chrząstowo, $9 \mathrm{~km}$ na W od Człuchowa, SHGZCH, s. 37-38. 
[k. 3v] Stretzin ${ }^{21}$ hoth 84 tzinßhuben der ist 31 besatzt und 20 zcu tzinse komen und tzinset von der hube $14 \mathrm{sc}, 2$ huner. Summa $11 \mathrm{mr}$ und $16 \mathrm{sc} .{ }^{\mathrm{d}}$-Item 7 garten tzinsen zcu $8 \mathrm{sc}$ und 4 huner. Item 1 garte $1 \mathrm{f}, 4$ huner. Item 5 garten zcu 1/2 f. Item 3 kretzen $2 \mathrm{mr}, 12 \mathrm{sch}$ haber. Item vom Mockenhole ${ }^{22} 2 \mathrm{mr}^{-\mathrm{d}}$ Summa: 31 besatzt, 11 freye, 53 wuste. Item 5 sullen /-sc-/ tzinsen im [14]50 ten jore. Item $6 \mathrm{im}[14] 52^{\text {ten }}$ jore.

Rosenfeld ${ }^{23}$ hoth 62 tzinßhuben der ist 13 besatzt und $10 \mathrm{zcu}$ tzinse komen und tzinset von der hube $1 / 2 \mathrm{mr}, 2$ huner. Summa $5 \mathrm{mr}$. Item 2 kretzem itzlicher $3 \mathrm{f}, 1$ ganß, sint wuste. Summa: 13 besatzt, 3 freye, 49 wuste. Item 1 sal tzinsen im [14]50 $0^{\text {ten }}$ jore. Item $2 \mathrm{im}[14] 52^{\text {ten }}$ jore.

Prewschenwald ${ }^{24}$ hoth 61 tzinßhuben der ist 27 besatzt und 18 zcu tzinse komen und tzinset von der hube $1 / 2 \mathrm{mr}, 2$ huner. Item 2 kruge zcu $3 \mathrm{f}$, der eine ist wuste. Summa $10 \mathrm{mr}$ minus f. Summa: 27 besatzt, item 9 freye, item 34 sint wuste. Item 3 sullen tzinsen im [14] $48^{\text {ten }}$ jore. Item 3 sullen tzinsen im[14]49 $9^{\text {ten }}$. Item $3 \mathrm{im}[14] 52^{\text {ten }}$ jore.

Summa $26 \mathrm{mr}$ und $10 \mathrm{sc}$.

[k. 4r] Petirswald ${ }^{25}$ hoth 52 tzinßhuben der ist 26 besatzt und 16 zcu tzinse komen und tzinset von der hube $1 / 2 \mathrm{mr}, 2$ huner. Item 2 kretzem zcu $3 \mathrm{f}$, einer ist wuste. Summa $9 \mathrm{mr}$ minus $1 \mathrm{f}$. Summa: 26 besatzt, 10 freye, 26 wuste. Item 7 sullen tzinsen im [14] $48^{\text {ten }}$ jore. Item 3 im [14]51 $1^{\text {ten }}$ jore.

Bysschofßwald ${ }^{26}$ hoth 64 tzinßhuben der ist 33 besatzt und $20 \mathrm{zcu}$ tzinse komen und tzinset von der hube $16 \mathrm{sc}, 2$ huner. Item 2 kruge tzinsen $2 \mathrm{mr} /$-und sullen geben-/. Summa $15 \mathrm{mr}$ und $8 \mathrm{sc}$. Summa: 33 besatzt, Item 31 wuste,

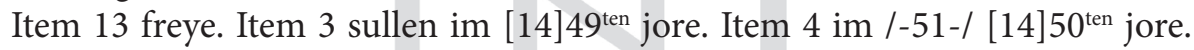
Item $6 \mathrm{im}[14] 51^{\text {ten }}$ jore.

Ffurstenaw ${ }^{27}$ hoth 60 tzinßhuben der ist 32 besatzt und 20 zcu tzinse komen und tzinset von der hube $14 \mathrm{sc}, 2$ huner. Summa $11 \mathrm{mr}$ und $16 \mathrm{sc}$. Item 3 kretzem itzlicher $1 \mathrm{mr}$ und $8 \mathrm{sc}$, sint wuste. Item 4 garten zcu $4 \mathrm{sc}$, sint wuste,

d--d Po prawej stronie uwaga: sint wuste.

${ }^{21}$ Strzeczona, $4 \mathrm{~km}$ na N od Debrzna, SHGZCH, s. 110-112.

${ }^{22}$ Mockenhole, w 1379 r. połowę dóbr rycerskich (gut Muckenhol) nadano sołtysowi i chłopom z sąsiedniej wsi Barkowo za roczny czynsz 2 grz., Handfesten der Komurei Schlochau, hrsg. v. Paul Panske, Danzig 1921 (dalej cyt. HKS), nr 124, s. 137. Dobra te znajdowały się zapewne między Barkowem i Strzeczoną.

${ }^{23}$ Rozwory, 8 km na W od Debrzna, SHGZCH, s. 101-102.

${ }^{24}$ Prusinowo Człuchowskie, $11 \mathrm{~km}$ na W od Debrzna, SHGZCH, s. 96-97.

${ }^{25}$ Cierznie, $10 \mathrm{~km}$ na NW od Debrzna, SHGZCH, s. 39-40.

${ }^{26}$ Biskupnica, 9 km na W od Człuchowa, SHGZCH, s. $24 \mathrm{v} 25$.

${ }^{27}$ Gwieździn, 12 km na NW od Człuchowa, SHGZCH, s. 59-60. 
und 2 huner. Summa: 32 besatzt, 20 tzinsen, 12 freye, 28 wuste. Item 3 sullen

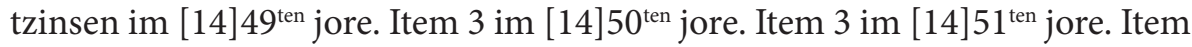
$3 \mathrm{im}[14] 52^{\text {ten }}$ jore.

Summa $35 \mathrm{mr}$ und $3 \mathrm{f}$.

[k. 4v] Stegirßwald ${ }^{28}$ hoth 80 tzinßhuben der ist/-32-/ 45 besatzt und $36 \mathrm{zcu}$ tzinse komen und tzinset von der hube $4 \mathrm{sc}^{29}$ Item 3 kruge sulden tzinsen itzlicher $2 \mathrm{mr}$ minus $8 \mathrm{sc}$, zo tzinsen sey 2 1/2 mr. Summa 23 1/2 mr. Summa: 36 tzinsen, 9 freye, 35 wuste. Item 4 huben sullen tzinsen in [14]49 ${ }^{\text {ten }}$ jore. Item $5 \mathrm{im}[14] 52^{\text {ten }}$ jore.

Hanßfeld ${ }^{30}$ hoth 60 tzinßhuben der ist 45 besatzt und 20 zcu tzinse komen und tzinset von der hube $14 \mathrm{sc}, 2$ huner. Summa $11 \mathrm{mr}$ und $16 \mathrm{sc}$. Item 2 kretzem itzlicher $1 \mathrm{mr}$ und $8 \mathrm{sc}$, sint wuste. Summa: 45 besatzt, 20 tzinsen, 25 freye,

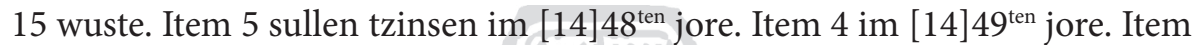
$6 \mathrm{im}[14] 52^{\text {ten }}$ jore.

Dempslaw $^{31}$ hoth 47 tzinßhuben der ist 25 besatzt und $17 \mathrm{zcu}$ tzinse komen und tzinset von der hube $11 \mathrm{sc}, 2$ huner. Summa $7 \mathrm{mr}$ und 19 sc. ${ }^{\mathrm{e}}$-Item 47 garten zcu $1 \mathrm{f}$. Item $1 \mathrm{mr}$ von kretzem. Item $3 \mathrm{f}$ von $\mathrm{kretzem}^{-\mathrm{e}}$. Summa: 25 besatzt, 17 tzinsen, 8 freye, 22 wuste. Item 3 huben tzinsen im [14] $48^{\text {ten }}$ jore. Item $5 \mathrm{im}$ $[14] 49^{\text {ten }}$ jore.

Summa 53 mr minus 1 sc.

[k. 5r] Valkenwald ${ }^{32}$ hoth 61 tzinßhuben der ist $271 / 2$ besatzt und 16 zcu tzinse komen und tzinset von der hube $14 \mathrm{sc}$. Summa $7 \mathrm{mr}$ und $19 \mathrm{sc}$. Item 2 kretzem itzlicher $1 \mathrm{mr}$, sint wuste. f-Item 1 garte $1 / 2 \mathrm{mr}$. Item 1 garte $8 \mathrm{sc}$. Item 1 garte 7 sc. $^{-f}$ Summa: $271 / 2$ besatzt, 16 tzinsen, $111 / 2$ freye, $221 / 2$ wuste.

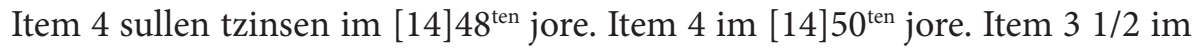
$[14] 52^{\text {ten }}$ jore

Grunaw $^{33}$ hoth 72 tzinßhuben der ist 25 besatzt und 11 zcu tzinse komen und tzinset von der hube $8 \mathrm{sc}$. Item 3 kretzem itzlicher $1 \mathrm{mr}, 2$ sint wuste. Summa

e--e $Z$ prawej strony uwaga: sint wuste.

f-f $Z$ prawej strony uwaga: sint wuste.

${ }^{28}$ Rzeczenica, $23 \mathrm{~km}$ na NE od Czarnego, SHGZCH, s. 103-104.

${ }^{29}$ Zapewne błąd pisarza. Czynsz z łanu wynosił 14 sc, co wynika z podanego sumowania. Taki sam czynsz z łanu pobierano w 1437 r., zob. Das grosse Zinsbuch, s. 109.

${ }^{30}$ Nadziejewo, $4 \mathrm{~km}$ na E od Czarnego, SHGZCH, s. 82.

${ }^{31}$ Domisław, $6 \mathrm{~km}$ na SE od Czarnego, SHGZCH, s. 53.

${ }^{32}$ Sokole, $3 \mathrm{~km}$ na SE od Czarnego, SHGZCH, s. 108-109.

${ }^{33}$ Stare Gronowo, pow. człuchowski, gm. Debrzno. Wieś w Królestwie Polskim od 1397 r., zastawione zakonowi krzyżackiemu, zob. M. Biskup, Wykaz krzywd i strat, s. 122, przyp. 99. Inaczej M. GrZEgorZ w: SHGZCH, s. 58. 
$4 \mathrm{mr}$ und $16 \mathrm{sc}$. Summa: 25 besatzt, 11 tzinsen, 14 freye, 47 wuste. Item 6 sullen tzinsen im [14] $48^{\text {ten }}$ jore. Item 5 sullen tzinsen im [14]51 $1^{\text {ten }}$ jore.

Prachlaw ${ }^{34}$ hoth 49 tzinßhuben der ist 44 besatzt und 32 zcu tzinse komen und tzinset von der hube $14 \mathrm{sc}, 2$ huner. Item 4 kretzem itzlicher $20 \mathrm{sc}$, einer ist wuste. Summa $21 \mathrm{mr}$ und 4 sc. Summa: 44 /-51-/ besatzt, 32 tzinsen, 5 wuste,

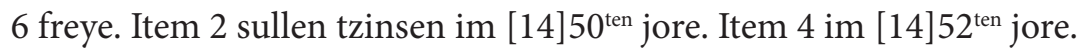

Flyssenstein ${ }^{35}$ hoth 60 tzinßhuben der ist 12 besatzt und $10 \mathrm{zcu}$ tzinse komen und tzinset von der hube $8 \mathrm{sc}, 2$ huner. Summa $3 \mathrm{mr}$. Item 3 kretzmen itzlicher $3 \mathrm{f}$, sint wuste. Item 12 besatzt, 10 tzinsen, 2 freyen, 48 /-51-/ wuste. Item 2 huben sullen tzinsen im [14]50 ${ }^{\text {ten }}$ jore.

Summa 36 und 15 sc.

[k. 5v] Sampoln ${ }^{36}$ hoth $301 / 2$ tzinßhuben der ist 11 besatzt und $8 \mathrm{zcu}$ tzine komen und tzinset von der hube $1 / 2 \mathrm{mr}, 2$ huner. Item 3 kretzem, 2 sint wuste, der eine tzinset $1 \mathrm{mr} 2 \mathrm{sc}$. Summa $5 \mathrm{mr} 2 \mathrm{sc}$. Item 2 garten itzlicher $8 \mathrm{sc}$, 2 huner, sint wuste. Summa: 11 besatzt, 8 tzinsen, 3 freye, 19 1/2 wuste. Item 3 sullen tzinsen im [14]49 $9^{\text {ten }}$ jore.

Newgut $^{37}$ hoth 44 tzinßhuben der ist 15 besatzt und ${ }^{\mathrm{g}-10}$ tzinsen $^{-\mathrm{g}}$ tzinset von der hube $1 / 2 \mathrm{mr}$. Summa $5 \mathrm{mr}$. Item 1 kretzem $1 \mathrm{mr}$, ist wuste. Summa: 15 besatzt, 10 tzinsen, 5 freye, 28 wuste. Item 1 sal tzinsen im [14] $48^{\text {ten }}$. Item $2 \mathrm{im}$ [14]49 $9^{\text {ten }}$ jore.

Summa 10 mr. 2 sc.

Summa der huben dy gelt pfleen zcu tzinsen 1473. Item do von is ir besatzt 735. Item /-5-/ 490 sint zcu tzinse komen. Item 246 sint noch frey. Item 594 $1 / 2$ sint wuste.

[k. 6r] Dye stete

Slochow ${ }^{38}$ Gebit $2 \mathrm{mr}$ und $9 \mathrm{~d}$ von den garten. Item $2 \mathrm{mr}$ von hokenbuden. Item $1 / 2 \mathrm{mr}$ vom meltzhawse, ist wuste. Summa $4 \mathrm{mr}$ und $9 \mathrm{~d}$

Conitz $^{39}$ Item alle jor 10 schyffpfund speckfleysch awssim saltze. Item 54 steyn unslet. Item dy becker alle jor $12 \mathrm{lb}$ pfeffir. Item $71 / 2 \mathrm{mr}$ alle quatemper. Item

g--g Tekst nadpisany ze znakiem wstawienia.

${ }^{34}$ Przechlewo, $15 \mathrm{~km}$ na NW od Człuchowa, SHGZCH, s. 97-99.

${ }^{35}$ Koczała, $15 \mathrm{~km}$ na E od Białego Boru, SHGZCH, s. 67-68.

${ }^{36}$ Sąpolno Człuchowskie, 15 km na N od Człuchowa, SHGZCH, s. 104-105.

${ }^{37}$ Nowa Wieś Człuchowska, 20 km na N od Człuchowa, SHGZCH, s. 86.

${ }^{38}$ Człuchów, miasto.

${ }^{39}$ Chojnice, miasto. 
dy walkmole hoth dys jor gegeben $12 \mathrm{mr}$ doch zo gebit sey ein jor mee wen daß andir.

Fredeland ${ }^{40}$ hoth 90 erbe, der ist 46 besatzt und $43 \mathrm{zcu}$ tzinse komen und tzinset vom erbe $5 \mathrm{sc}$ und $10 \mathrm{~d}$. Item itzlich erbe gebit $6 \mathrm{~d}, 1 / 2$ erbe $3 \mathrm{~d}$, das heyst strossen geld.

Summa $10 \mathrm{mr} 4 \mathrm{sc}$ minus $4 \mathrm{~d}$.

Summa $61^{\text {h- }} \mathrm{mr}^{-\mathrm{h}} 4 \mathrm{sc}$ und $5 \mathrm{~d}$.

[k. $6 v$ ] Hamersteyn ${ }^{41}$ hoth 11 1/2 hube und $161 / 2$ morgen und gebit von der hube /-15-/ 14 sc.

Item hoth 43 morgen krug land do von gebit sey 9 f 3 sc.

Item $1 \mathrm{mr}$ und 4 1/2 sc von hokenbuden und brotbrenken [s].

Summa $10 \mathrm{mr} 5 \mathrm{sc}$ und $3 \mathrm{~d}$.

Baldenburg ${ }^{42}$ hoth dyß jor getzinset $31 / 2 \mathrm{mr}$ und sulde wol tzinsen $41 / 2 \mathrm{mr}$ sundir ist wuste.

Item dy sneydemole zcu Flyssenstein gebit $6 \mathrm{mr}$.

Swen und ku tzinß

Sichtze $^{43} 3$ f, Czoßnaw ${ }^{44} 11 / 2$ mr, Pransen ${ }^{45} 11 / 2$ mr, Ratelwitz ${ }^{46} 1 / 2 \mathrm{mr}$, Re$\operatorname{dern}^{47} /-4$ mr-/ 6 mr, Gleysten ${ }^{48} 11 / 2$ mr, Sukaw ${ }^{49} 21 / 2$ mr

Summa 12 mr und $1 \mathrm{f}$

${ }^{\mathrm{i}-} \mathrm{Qwarsin}{ }^{50} 3 \mathrm{mr}, \mathrm{Czyten}^{51} 2 \mathrm{mr}^{-\mathrm{i}}$

Summa $32 \mathrm{mr}$ minus $1 \mathrm{sc}$

h- -h Tekst nadpisany.

${ }^{\mathrm{i}-\mathrm{i}} Z$ prawej strony uwaga: wuste.

${ }^{40}$ Debrzno, miasto.

${ }^{41}$ Czarne, miasto.

${ }^{42}$ Biały Bór, miasto.

${ }^{43}$ Żychce, $20 \mathrm{~km}$ na N od Człuchowa, SHGZCH, s. 120-121.

${ }^{44}$ Czosnowo, około $16 \mathrm{~km}$ na $\mathrm{N}$ od Człuchowa, SHGZCH, s. 47.

${ }^{45}$ Prądzona, $21 \mathrm{~km}$ na S od Bytowa, SHGZCH, s. 96.

${ }^{46}$ Racławki, 7 km na E od Chojnic, Maksymilian Grzegorz, Słownik historyczno-geograficzny komturstwa tucholskiego w średniowieczu, Tuchola-Bydgoszcz 2010, s. 90-91.

${ }^{47}$ Redern, wieś rycerska zaginiona, SHGZCH, s. 100.

${ }^{48}$ Gliśno, 13 km na SW od Bytowa, SHGZCH, s. 57.

${ }^{49}$ Żukowo, 14 km na E od Chojnic, SHGZCH, s. 121.

${ }^{50}$ Qwarsin, osada zaginiona, SHGZCH, s. 99.

${ }^{51}$ Szczytno, 14 km na NW od Człuchowa, SHGZCH, s. 113-114. 
[k. $7 r]$ Mole tzinß alle quatemper

Wintmole zcu Slochow ${ }^{52}$ tzinset $3 \mathrm{f}$, Fredeland ${ }^{53}$ hoth getzinset $121 / 2 \mathrm{mr}$ und tzinset 4 1/2, Hamersteen ${ }^{54}$ hoth tzinset $15 \mathrm{mr} /-1$-/ und tzinset 2 1/2, Sprynckmole ${ }^{55}$ tzinset $1 \mathrm{mr}$, Puschmole ${ }^{56}$ hotz tzinset $5 \mathrm{f}$ und tzinset $1 \mathrm{mr}$, Hanßmole ${ }^{57}$ hoth getzinset $21 / 2 \mathrm{mr}$ tzinset $1 \mathrm{mr}$, Stegir $\beta^{58}$ hoth getzinset $7 \mathrm{f}$ tzinset $1 \mathrm{mr}$, Funckenmole ${ }^{59}$ tzinset $2 \mathrm{mr}$, Qweßdin ${ }^{60}$ tzinset $3 \mathrm{f}$ alle quatemper.

Summa alle quatemper $15 \mathrm{mr}$ macht daß jor $60 \mathrm{mr}$

${ }^{j}$-Polnitz 9 f quatemper, Czyremole ${ }^{61} 5$ f quatemper, Bysschofswald $1 \mathrm{mr}$, Czyten $^{62} 11 / 2 \mathrm{mr}$, wintmole zcu Reychenaw ${ }^{63} 3 \mathrm{f}$, Brenßmole ${ }^{64} 5 \mathrm{mr}$, Cristfeld $11 / 2 \mathrm{mr}$, Petirßwaldeschemole ${ }^{65} \mathrm{mr}^{-\mathrm{j}}$

Summa summarum alleß geldis gelde daß itzund gefeld awß steten, dorffern, molen molen [s] polenschen guttern und von alle gutter tzinsen ist $441 \mathrm{mr}$ minus $8 \mathrm{~d}$ guden gelde

[k. $7 v]$ Deße nochgeschrebin stete und dorffir tzinsen getreyde

Conitz $^{66}$ hoth hundert huben der ist 80 besatzt und zcu tzinse komen und tzinset von der hube 2 sch weyse, 3 sch rocken, 3 sch gerste, 2 sch haber und 1 ganß. Summa: 2 leste und $40 \mathrm{sch}$ weyse, 2 leste und 40 sch haber, 4 leste rokken, 4 leste gerste.

Fredeland ${ }^{67}$ hoth 86 tzinßhuben der ist 80 besatzt und $70 \mathrm{zcu}$ tzinse komen und tzinset von der hube 3 sch rocken, 3 sch gerste, 2 sch haber. Summa: 3 1/2 last rocken, 3 1/2 last gerste, 2 lest und 20 sch haber. Summa: 80 besatzten huben, 70 tzinsen, 10 freye, 6 wuste. Item 10 freye sullen tzinsen im [14]51 ${ }^{\text {ten }}$ jore.

j--j Z prawej strony uwaga: sint wuste.

${ }^{52}$ Człuchów wiatrak.

${ }^{53}$ Debrzno.

${ }^{54}$ Czarne.

${ }^{55}$ Sprynckmole, R. KuBICKI, op.cit., s. 509.

${ }^{56}$ Buschmühle, młyn na południe od leśniczówki Wolność i na zachód od wsi Charzykowy, R. KuвICKI, op.cit., s. 536.

${ }^{57}$ Hanßmole, R. Kuвicki, op.cit., s. 449.

${ }^{58}$ Rzeczenica, $23 \mathrm{~km}$ na NE od Czarnego, SHGZCH, s. 103-104.

${ }^{59}$ Funka (niem. Funker Mühl) koło wsi Charzykowy, R. Kubicki, op.cit., s. 443.

${ }^{60}$ Brzeźno Szlacheckie, 30 km na NE od Białęgo Boru, SHGZCH, s. 28-29.

${ }^{61}$ Prądy koło wsi Lędyczek (15 km na W od Debrzna), R. Kubicki, op.cit., s. 495-496.

${ }^{62}$ Szczytno, R. Kubicki, op.cit., s. 519.

${ }^{63}$ Rychnowy wiatrak.

${ }^{64}$ Brenßmole, R. Kubicki, op.cit., s. 429.

${ }^{65}$ Młyn Nierybie część sołectwa Cierznie.

${ }^{66}$ Chojnice.

${ }^{67}$ Debrzno. 
Slochow ${ }^{68}$ hoth 12 1/2 halbe tzinßhuben und sint besatzt und tzinset von der hube 4 sch rocken, 4 sch gerste, 4 sch haber, 2 huner. Summa: 50 sch rocken, $50 \mathrm{sch}$ gerste, $50 \mathrm{sch}$ haber.

Buchholtz ${ }^{69}$ hoth 61 tzinßhuben der ist 22 besatzt und $17 \mathrm{zcu}$ tzinse komen und tzinset von der hube 5 sch rocken, 5 sch gerste, 9 sch haber, 2 huner. Summa: 1 last und 25 sch rocken, 1 last und 25 sch gerste, 2 1/2 last und 3 sch haber. Summa: 22 besatzt, 17 tzinsen, 41 wuste, 5 freye. Item 2 sullen tzinsen im

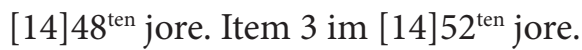

[k. 8r] $\operatorname{Mossin}^{70}$ hoth 63 tzinßhuben der ist 50 besatzt, der $40 \mathrm{zcu}$ tzinse komen und tzinset von der hube 5 sch rocken, 5 sch gerste und 9 sch haber und 2 huner. Summa: 3 leste und $20 \mathrm{sch}$ rocken, 3 leste und $20 \mathrm{sch}$ gerste, 6 leste haber. Summa: /-40-/ 50 huben besatzt, 40 tzinsen, 13 wuste, 10 freye. Item 2 sullen

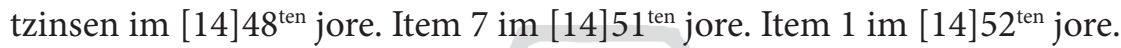

Marienfeld ${ }^{71}$ hoth 31 tzinßhuben der ist 10 besatzt und zcu tzinse komen und tzinset von der hube 4 sch rocken, 4 gerste, 6 sch haber, 2 huner. Summa: $40 \mathrm{sch}$ rocken, $40 \mathrm{sch}$ gerste, 1 last haber. Item 21 wuste huben.

Heynrichswald ${ }^{72}$ hoth 90 tzinßhuben der 29 besatzt und 27 zcu tzinse komen und tzinset von der hube $4 \mathrm{sch}, 4 \mathrm{sch}$ [s] gerste, $4 \mathrm{sch}$ haber, 2 huner. Summa: 1 last und 48 sch rocken, 1 last und 48 sch gerste, 1 last und 48 sch haber. Summa: 29 besatzten, 27 tzinsen, 2 freye, 61 wuste. Item 1 sal tzinsen im [14]48 ${ }^{\text {ten }}$. Item $1 \mathrm{im}[14] 50^{\text {ten }}$ jore.

[k. $8 v$ ] Birkenfeld ${ }^{73}$ hoth 62 tzinßhuben der 24 besatzt und $17 \mathrm{zcu}$ tzinse komen und tzinset von der hube 4 sch rocken, 4 sch gerste, 4 sch haber, 2 huner. Summa: 1 last und $8 \mathrm{sch}$ rocken, 1 last und $8 \mathrm{sch}$ gerste, 1 last und $8 \mathrm{sch} /$-gerste-/ haber. Summa: 24 besatzten huben, 17 tzinsen, 38 sint wuste, 7 freye. Item

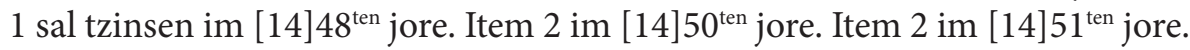
Item $2 \mathrm{im}[14] 52^{\text {ten }}$ jore.

Crystfeld ${ }^{74}$ hoth 61 tzinßhuben der ist 22 besatzt und 21 zcu czinse komen und tzinset von der hube 2 sch rocken, 3 sch gerste, 4 sch haber. Summa: $42 \mathrm{sch}$ rocken, 1 last und 3 sch gerste, 1 last und 24 sch haber. Summa: 22 besatzt, 21 tzinsen, 1 freye, 39 wuste. Item 1 hube sal tzinsen im [14]51 $1^{\text {ten }}$ jore.

\footnotetext{
${ }^{68}$ Człuchów.

${ }^{69}$ Bukowo Człuchowskie, $8 \mathrm{~km}$ na S od Człuchowa, SHGZCH, s. 29-30.

${ }^{70}$ Mosiny, $7 \mathrm{~km}$ na S od Człuchowa, SHGZCH, s. 79-80.

${ }^{71}$ Myśligoszcz, 5 km na NE od Debrzna, SHGZCH, s. 81.

${ }^{72}$ Uniechów, 8 km na NW od Debrzna, SHGZCH, s. 116-117.

${ }^{73}$ Barkowo, $12 \mathrm{~km}$ na SW od Człuchowa, SHGZCH, s. 19-20.

${ }^{74}$ Chrząstowo, $9 \mathrm{~km}$ na SW od Człuchowa, SHGZCH, s. 37-38.
} 
Cramptzk ${ }^{75}$ hoth 50 tzinßhuben der ist / $23-$ / $^{k}-33^{-k}$ besatzt und 27 zcu tzinse komen und tzinset von der hube: 4 sch rocken, 4 sch gerste, 4 sch haber, 2 huner. Summa: 1 last und $48 \mathrm{sch}$ rocken, 1 last und 48 sch gerste, 1 last und $48 \mathrm{sch}$ haber. Summa: 33 besatzt, 27 tzinsen, 6 freye, 17 wuste. Item 3 huben sullen

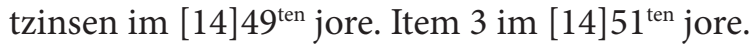

[k. 9r] Summa der huben dy getreyde pfleen zcu tzinsen 605 1/2 huben. Item 362 1/2 sint besatzt. Item 321 1/2 sint zcu tzinse komen. Item 2 sint noch frey. Item 245 sint noch wuste.

Summa deß rocken von den huben. 18 leste und 41 1/2 sch rocken. Item 19 leste $32 \mathrm{sch}$ gerste. Item 20 leste $41 \mathrm{sch}$ haber. Item 2 leste und $40 \mathrm{sch}$ weyse.

Item molen tzinsß

Dunkelßhagin ${ }^{76}$ tzinset alle jor 6 leste rocken und 6 leste maltz. Virchow /-tzin-/ sulde tzinsen 8 leste mel und tzinset /-4-/ 3 leste meel. Blumenfeld ${ }^{77}$ sulde tzinsen, leyt wuste. Petirwald $51 / 2$ rocken ist wuste.

Summa awssm molen 9 leste rocken 6 leste maltz.

Item zo ist diß jor gefallen von der landmitte ${ }^{1 /} /$ und awssm..........- $/^{-1} 10$ leste rocken

Summa alliß rocken 37 leste rocken ${ }^{\mathrm{m}}$ und $^{-\mathrm{m}} 41$ 1/2 sch rocken

Item zo ist diß jor gefallen 3 leste gerste von der landmitte

Summa allir gerste 22 leste und 32 sch gerste und 6 leste maltz

Item zo ist diß jor gefallen von inen frawenhabir ${ }^{78}$ und walthaber 11 leste haber Item landmitte 8 leste haber

Summa allis habirn der dyß jor ist gefallen 39 leste

[k. 9v] Summa summarum alliß getreydeß an tzinsern, molen, steten, dorffern, landmitte, inen frawenhabir und allem getreyde ${ }^{n-}{ }^{n} \beta^{-n}$ gefallen ist hundert leste und 7 leste und 21 sch.

Summa allir huben 1 dye pfleen zcu tzinsen obir 273 1/2 huben.

${ }^{\mathrm{k}-\mathrm{k}}$ Tekst nadpisany.

${ }^{1--1}$ Wykreślony tekst nieczytelny.

$\mathrm{m}-\mathrm{m}$ Tekst nadpisany.

${ }^{\mathrm{n}-\mathrm{n}}$ Tekst nadpisany.

${ }^{75}$ Krępsk, 9 km na NW od Człuchowa, SHGZCH, s. 71-72.

${ }^{76}$ Tunkiel, wieś włączona do m. Chojnic, SHGZCH, s. 115.

${ }_{77}^{71}$ Niwy, 6 km od Kamienia, SHGZCH, s. 83-84.

${ }^{78}$ Różne hipotezy odnośnie znaczenia tej daniny zob. HKS, s. 211-213. 
Item der ist obir ab besatzt 1097 1/2. Item der ist 811 1/2 zcu tzinse komen. Item 297 haben noch freyheyt. Item der ist 976 wuste.

Item zo ist diß jor gefallen 34 tone honige der ist ken Marienburg ${ }^{79}$ gekomen 16. Ouch zo gefelt ein jor mer wen daß andir.

[k. 10r] [karta pusta]

[k. 10v] Dy dinste

${ }^{0-}$ Gerhartßdorf $^{80}$, Geylenfeld ${ }^{81}$, Gottzendorff ${ }^{82}$, Butzkendorff ${ }^{83}$, Gezeryk ${ }^{84}$, Moßnytz $^{85}$, Jocubßdorff ${ }^{86}$, Stolzenfeld ${ }^{87}$, Berenwald ${ }^{88}$, Byrgelaw ${ }^{89}$, Sbenyn ${ }^{90}$, Platendinst $^{91}$, Conarsyn ${ }^{92}$, Breytenfeld ${ }^{93}$, Lancke ${ }^{94}$, Lozyn ${ }^{95}$, Krummensehe ${ }^{96}$, Dinkerßhagin $^{97}$, Borsekow ${ }^{98}$, Czyten, Gotzkow, Doringeßdorff ${ }^{99}$, Glanden ${ }^{100}$, Dompsla Brae ${ }^{101}$, Schonewerdir ${ }^{102}$, Cleyne Gentznik ${ }^{103}$, Clawßfeld ${ }^{104}$, Dame-

\footnotetext{
${ }^{79}$ Malbork.

${ }^{80}$ Ogorzeliny, 7 km na N od Kamienia, SHGZCH, s. 88-89.

${ }^{81}$ Wyczechy, 6 km na E od Czarnego, SHGZCH, s. 118.

${ }^{82}$ Gockowo, $9 \mathrm{~km}$ na NE od Czarnego, SHGZCH, s. 57.

${ }^{83}$ Objezierze, $11 \mathrm{~km}$ na E od Chojnic, SHGZCH, s. 87.

${ }^{84}$ Jeziorki, $9 \mathrm{~km}$ na E od Chojnic, SHGZCH, s. 63.

${ }^{85}$ Moszczenica, 7 km na SW od Chojnic, SHGZCH, s. 80-81.

${ }^{86}$ Zamarte, 7 km na N od Kamienia, SHGZCH, s. 118-119.

${ }^{87}$ Stołczno, 6 km na NW od Człuchowa, SHGZCH, s. 110-112.

${ }^{88}$ Bińcze, 13 km na W od Człuchowa, SHGZCH, s. 24.

${ }^{89}$ Czarnoszyce, $10 \mathrm{~km}$ na NE od Człuchowa, SHGZCH, s. 43-44.

${ }^{90}$ Zbeniny, $6 \mathrm{~km}$ na NE od Chojnic, SHGZCH, s. 120.

${ }^{91}$ Płonica, $7 \mathrm{~km}$ na S od Człuchowa, SHGZCH, s. 93-94.

${ }^{92}$ Konarzyny, $17 \mathrm{~km}$ na N od Człuchowa, SHGZCH, s. 69-70.

${ }^{93}$ Sierpowo, $6 \mathrm{~km}$ na S od Czarnego, SHGZCH, s. 105-106.

${ }^{94}$ Łąkie, $20 \mathrm{~km}$ na S od Bytowa, SHGZCH, s. 76-77.

${ }^{95}$ Łoża, $9 \mathrm{~km}$ na E od Czarnego, SHGZCH, s. 77.

${ }^{96}$ Krzemieniewo, $9 \mathrm{~km}$ na S od Czarnego, SHGZCH, s. 72.

${ }^{97}$ Tunkiel, obecnie część miasta Chojnic, SHGZCH, s. 115.

${ }_{98}$ Borzyszkowy, $40 \mathrm{~km}$ na N od Człuchowa, SHGZCH, s. 26.

${ }^{99}$ Doręgowice, 9 km na SE od Człuchowa, SHGZCH, s. 54.

${ }^{100}$ Glandaw, dobra zaginione, SHGZCH, s. 55-56.

${ }^{101}$ Dompsla Brae (Thomyslaw by der Bra - Domysław nad Brdą), zaginione dobra rycerskie, położone nad rzeką Brdą (niem. Brahe), obok miejscowosci Lotyń i Żukowo, HKS, nr 109,

${ }^{102}$ Skowarnki, 10 km na NW od Debrzna, SHGZCH, s. 106.

${ }^{103}$ Jęczniki Małe, 5 km na SE od Człuchowa, SHGZCH, s. 63.

${ }^{104}$ Jaromierz, 5 km na SW od Człuchowa, SHGZCH, s. 61-62.
} s. $122-123$. 
raw $^{105}$, Tzelelaw ${ }^{106}$, Leypnitz ${ }^{107}$, Ratelwitz, Pawelkow ${ }^{108}$, Jocusch Conitz ${ }^{109}$, Lyssow $^{110}$, Elzenaw ${ }^{111}$, Sychtze, Gleysten, Qweßden ${ }^{112}$, Gemelen ${ }^{113}$, Pransen, Czoßnow $^{114}$, Prewschendorff ${ }^{115}$, Nezewantz ${ }^{116-0}$

\section{Summa 43}

[k. 11r] ${ }^{\mathrm{p}-S c h o n e n f e l d}{ }^{117}$, Hogenwald ${ }^{118}$, Sabyst ${ }^{119}$, Rawtenberg ${ }^{120}$, Blumenfeld, Hennygßdorff ${ }^{121}$, Crayanten ${ }^{122}$, Cladaw $^{123-p}$

${ }^{\mathrm{r}-G r o s s e}$ Pawlow $^{124}$, Pagdantz ${ }^{125}$, Hermanßdorff ${ }^{126}$, Osterwyk ${ }^{127}$, Brysnitz ${ }^{128}$, Lottin $^{129-r}$

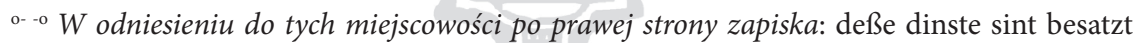
und dinen.

p--p W przypadku tych miejscowości z prawej strony zapisano: uff desen guttern wonen scholtzen.

${ }^{\mathrm{r}-\mathrm{r}}$ W przypadku tych miejscowości z prawej strony zapisano: Deße dinste habin dy erbar lewte awßgekowfft und scholtzen doruff gesatzt und dy dinen nicht alzo fulkomelichen alß unser scholtzen. ${ }^{105}$ Dąbrowa Człuchowska, $15 \mathrm{~km}$ na N od Człuchowa, SHGZCH, s. 47-48.

${ }^{106}$ Ciecholewy, 16 na N od Człuchowa, SHGZCH, s. 38-39.

${ }^{107}$ Lipnica, $20 \mathrm{~km}$ na S od Bytowa, SHGZCH, s. 73v74.

${ }^{108}$ Pawłówko, 9 km na NW od Człuchowa, SHGZCH, s. 92-93.

${ }^{109}$ Jocoschconitz, dobra rycerskie zaginione, obecnie część wsi Jarcewo, HKS, s. 101-104.

${ }^{110}$ Lisewo, $12 \mathrm{~km}$ na NW od Człuchowa, SHGZCH, s. 74-76.

${ }^{111}$ Olszanowo, $13 \mathrm{~km}$ na E od Czarnego, SHGZCH, s. 89.

${ }^{112}$ Gwiazda (niem. Gwesden, Gwesdin) dobra rycerskie, zaginione, SHGZCH, s. 59.

${ }^{113}$ Jemielno, $12 \mathrm{~km}$ na N od Człuchowa, SHGZCH, s. 62.

${ }^{114}$ Czosnowo, $16 \mathrm{~km}$ na $\mathrm{N}$ od Człuchowa, SHGZCH, s. 47.

${ }^{115}$ Preuschendorf, osada zaginiona położona koło wsi Niwy, SHGZCH, s. 96.

${ }^{116}$ Nieżywięć, $3 \mathrm{~km}$ na W od Chojnic, SHGZCH, s. 83.

${ }^{117}$ Nieżychowice, $3 \mathrm{~km}$ na SW od Chojnic. Jako Szenfeld zob. SHGZCH, s. 112.

${ }^{118}$ Hohenwalde, zaginione dobra rycerskie $10 \mathrm{~km}$ na W od Człuchowa, SHGZCH, s. 60.

${ }^{119}$ Czartołomie (w średniowieczu Zawist - Zawiść), obecnie część wsi Jarcewo, SHGZCH, s. $44,119-120$.

${ }^{120}$ Raciniewo, $7 \mathrm{~km}$ na E od Czarnego, SHGZCH, s. 99-100.

${ }^{121}$ Angowice, $4 \mathrm{~km}$ na S od Chojnic, SHGZCH, s. 19-20.

${ }^{122}$ Krojanty, $5 \mathrm{~km}$ na NE od Chojnic, SHGZCH, s. 72-73.

${ }^{123}$ Kłodawa, 6 km na NE od Chojnic, SHGZCH, s. 67.

${ }^{124}$ Pawłowo, $5 \mathrm{~km}$ na SE od Chojnic, SHGZCH, s. 91-92.

${ }^{125}$ Pakotulsko, 18 km na NW od Człuchowa, SHGZCH, s. 91.

${ }^{126}$ Jerzmionki, 8 km na N od Kamienia, SHGZCH, s. 62.

${ }^{127}$ Ostrowite, $18 \mathrm{~km}$ na S od Bytowa, SHGZCH, s. 90-91.

${ }^{128}$ Brzeźnica, $3 \mathrm{~km}$ na E od Białego Boru, SHGZCH, s. 27.

${ }^{129}$ Lotyń, 11 km na E od Chojnic, SHGZCH, s. 74-76. 
${ }^{\text {s- Grunßberg }}{ }^{130}$, Schonaw ${ }^{131}$, Everßfeld ${ }^{132}$, Demyn ${ }^{133}$, Schoneberg ${ }^{134}$, Heynrichßdorff $^{135}$, Dresen ${ }^{136}$, Grabaw ${ }^{137}$, Petirkow ${ }^{138}$, Maldyten $^{139}$, Ostatz ${ }^{140}$, Hulffe ${ }^{141}$, Remmen $^{142}$, Falkenhagin ${ }^{143}$, Reynenfeld ${ }^{144}$, Stremelow ${ }^{145}$, Ramkenßgut ${ }^{146-s}$

Summa 17

${ }^{\mathrm{t}-W a l t e r ß d o r f}{ }^{147}$, Polnitz, Fredeland ${ }^{148}$, Schiltberg ${ }^{149}$, Valkenwald, Rosenfeld ${ }^{-\mathrm{t}}$ [k. 11v] Scholtzen

Heynrychßwald, Lychtenhagen, Reychenow, Petirßwald, Rosenfeld, Birkenfeld, Valkenwald, Stretyn, Prechlaw, Crystfeld, /-Mie-/ Marienfeld, Buchholtz, Mossin, Gentznik, Dampnitz, Bysschofßwald, Hansfeld, /-Dampnitz-/, Stegers $^{150}$, Furstenaw, Mewßkendorff, Wenige Conitz, New Kirche, Brysen, Prewschenwald, Kramptzk, Virchow, Polnitz, Steynborn, Dompsla, Grunaw

Nadesłany 18 VII 2017

Nadesłany po poprawkach recenzyjnych 16 IX 2017

Zaakceptowany 24 IX 2017

Dr hab. Rafał Kubicki, prof. UG

Instytut Historii

Uniwersytet Gdański

e-mail: hisrk@univ.gda.pl

${ }^{\text {s-s }} Z$ prawej strony obok tych miejscowości zapisano uwagę: sint wuste.

${ }^{\mathrm{t}-\mathrm{t}} Z$ prawej strony obok tych miejscowości zapisano uwagę: Somerlinge.

${ }^{130}$ Klawkowo, na NE od Chojnic. Brak hasła w SHGZCH.

${ }^{131}$ Drzonowo, $8 \mathrm{~km}$ na S od Białego Boru, SHGZCH, s. 54-55.

${ }^{132}$ Przybyradz, 6 km na S od Białego Boru, SHGZCH, s. 99.

${ }^{133}$ Dyminek, $10 \mathrm{~km}$ na N od Czarnego, SHGZCH, s. 55.

${ }^{134}$ Kaliska, 4 km na NE od Białego Boru, SHGZCH, s. 64-65.

${ }_{135}$ Przeradz (niem. Heinrichsdorf), pow. bytowski, gm. Miastko, HKS nr 27, s. 40-42; nr 87, s. 101 .

${ }^{136}$ Dźwierzno, $15 \mathrm{~km}$ na NE od Białego Boru, SHGZCH, s. 55.

${ }^{137}$ Grabowo, 6 km na E od Białego Boru, SHGZCH, s. 57-58.

${ }^{138}$ Pietrzykowo, $24 \mathrm{~km}$ na NE od Białego Boru, SHGZCH, s. 93.

${ }^{139}$ Malditen, osada zaginiona położona koło wsi Niwy i Zamarte, SHGZCH, s. 77-78.

${ }^{140}$ Ostrza, $14 \mathrm{~km}$ na S od Czarnego, SHGZCH, s. 91.

${ }^{141}$ Pomoc (niem. Hülfe), część miasta Chojnic, SHGZCH, s. 61.

${ }_{142}$ Rębno, $5 \mathrm{~km}$ na NE od Lędyczka, SHGZCH, s. 100.

${ }^{143}$ Miłocice (niem. Falkenhagen), pow. bytowski. gm. Miastko.

${ }^{144}$ Barnowiec (niem. Reinfeld), pow. bytowski, gm. Miastko.

145 Trzemielewo, $4 \mathrm{~km}$ na SE od Białego Boru, SHGZCH, s. 115.

${ }^{146}$ Ramkensguth zaginione dobra rycerskie.

${ }^{147}$ Kiełpin, 5 km na NE od Człuchowa, SHGZCH, s. 66-67.

${ }^{148}$ Debrzno.

${ }^{149}$ Kamień, 7 km na N od Debrzna, SHGZCH, s. 65.

${ }^{150}$ Rzeczenica, 23 km na NE od Czarnego, SHGZCH, s. 103-104. 


\section{Register of Rents and Services of the Commandry \\ of CzŁuchów from the Year 1446 Along With an Inventory \\ of Horses and Kitchen Supplies in CzŁuchów Castle}

\section{Summary}

Key words: the Teutonic Order, rural economy, Eastern Pomerania

The edition presented here encompasses the register of rents and services of the commandry of Człuchów written in 1446, now preserved in the Secret State Archives Prussian Cultural Heritage (Geheimes Staatsarchiv Preußischer Kulturbesitz) in Berlin Dahlem. It also includes the inventory of horses, weapons and kitchen supplies in Człuchów castle, which was published earlier in the so-called Great Book of Offices (Das grosse Ämterbuch des Deutschen Ordens).

The register of rents from the year 1446 is similar in content to the register of rents of the commandry from the year 1437 published as part of the so-called Great Rent Book (Das grosse Zinsbuch des Deutschen Ritterordens), including the data acquired during a nationwide visit. The register of 1446 includes more detailed information about the subject matter, as it records not only the number of hinds actually populated along with the value of the rent paid for them, but also the number of empty hinds and hinds in the process of settlement (in the period of the so-called wolnizna) with the date of the payment of the first rent. The register also includes rents imposed on inns and mills operating in the territory of the commandry, the former payments of knights according to Polish law (the monetary equivalent of a cow and a pig - the payment was referred to as swen und ku tzinß) along with the so-called forest oat (walthaber) - the charge for permission to collect brushwood and pasture the cattle in the beech forests of the Teutonic Order. In the final part, the source includes the register of the knight's servants along with a list of estates that were manned with peasants or had a military function as well as abandoned ones, unable at that time to play any military role.

Das Verzeichnis der Gebühren und Dienste in Der Komturei Schlochau Aus DEm Jahr 1446 Und Das Inventar AN Pferden, Waffen und Küchenvorräten in der Burg von Schlochau

\section{Zusammenfassung}

Schlüsselwörter: Deutscher Orden, Landwirtschaft, Pommerellen

Das Verzeichnis der Gebühren und Dienste in der Komturei Schlochau aus dem Jahr 1446 und das Inventar an Pferden, Waffen und Küchenvorräten in der Burg von Schlochau

Die vorgestellte Edition umfasst ein 1446 hergestelltes Verzeichnis der Gebühren und Dienste in der Komturei Schlochau, das gegenwärtig im Geheimen Staatsarchiv 
Preußischer Kulturbesitz in Berlin-Dahlem aufbewahrt wird. Berücksichtigt wurde auch das an seinem Anfang befindliche Inventar von Pferden, Waffen und Küchenvorräten in der Burg in Schlochau, dass schon früher im sogenannten „Großen Ämterbuch des Deutschen Ordens") publiziert worden ist.

Das Verzeichnis der Gebühren von 1446 ähnelt in seinem Inhalt einer Zusammenstellung der Gebühren der Komturei, die im sogenannten „Großen Zinsbuch des Deutschen Ritterordens" publiziert ist und die in Wirklichkeit Angaben enthält, die im Rahmen eine Landesvisitation gewonnen wurden. Dabei überliefert das Verzeichnis von 1446 normalerweise genauere Angaben zu diesem Thema, denn neben der Gesamtzahl des zinsbaren Grundes vermerkt es nicht nur die Zahl der tatsächlich besiedelten Hufen zusammen mit der Höhe des von ihnen bezahlten Zinses, sondern auch die Zahl der brachliegenden Hufen und derer, die erneut bebaut wurden (in der Periode der Steuerfreiheit), einschließlich Angabe des Termins für die Zahlung des ersten Zinses. In dem Verzeichnis sind auch die Zinszahlungen berücksichtigt, die von den Schenken und Mühlen erhoben wurden, die auf dem Gebiet der Komturei betrieben wurden, ebenso die ehemaligen Leistungen der Rittergüter zu polnischem Recht, die in Zinszahlungen umgewandelt worden waren (das Äquivalent für eine Kuh und ein Schwein in Geld, die Gebühr wurde als swen und ku tzin $\beta$ bezeichnet) sowie der sogenannte Waldhafer (walthaber), eine Gebühr für die Erlaubnis zum Sammeln von Reisig und Viehfutter in den Buchenwäldern des Ordens. In ihrem letzten Teil enthält die besprochene Quelle eine Zusammenstellung der Ritterdienste, in dem sowohl die Rittergüter erwähnt werden, die mit Bauern besetzt waren und Heeresfolge leisteten, als auch die verwaisten, die zu dieser Zeit nicht in der Lage waren ihre militärischen Verpflichtungen zu erfüllen.

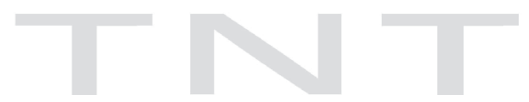


Wykaz czynszów i służb komturstwa człuchowskiego z 1446 roku...

\section{BiBLIOGRAFIA}

Biskup, Marian. „Skład konwentu zakonu krzyżackiego w Człuchowie z 1413 r.”. Zapiski Historyczne 63/1 (1998): 123-128.

Biskup, Marian. „Wykaz krzywd i strat wyrządzonych archidiecezji gnieźnieńskiej w okręgach człuchowskim i tucholskim z roku 1436". Zapiski Historyczne 69/1 (2004): 111-123.

Bruski, Klemens. Lokalne elity rycerstwa na Pomorzu Gdańskim w okresie panowania zakonu krzyżackiego studium prozopograficzne. Gdańsk: Wydawnictwo Uniwersytetu Gdańskiego, 2002.

Grzegorz, Maksymilian. Słownik historyczno-geograficzny komturstwa tucholskiego w średniowieczu. Tuchola: Starostwo Powiatowe; Bydgoszcz: Wydawnictwo UKW, 2010.

Grzegorz, Maksymilian. Słownik historyczno-geograficzny ziemi chojnickiej w granicach komturstwa człuchowskiego. Chojnice: CTPN, 2005.

Joachim, Erich, Walther Hubatsch, eds. Regesta historico-diplomatica Ordinis S. Mariae Theutonicorum 1198-1525, part 1, vol. 3: (1511-1525). Göttingen: Vandenhoeck \& Ruprecht, 1973.

Joachim, Erich, Walther Hubatsch, eds. Regesta historico-diplomatica Ordinis S. Mariae Theutonicorum 1198-1525, part 1, vol 1: (1198-1454). Göttingen: Vandenhoeck \& Ruprecht, 1948.

Kubicki, Rafał. Młynarstwo w państwie zakonu krzyżackiego w Prusach w XIII-XV wieku (do 1454 r.). Gdańsk: Wydawnictwo Uniwersytetu Gdańskiego, 2012.

Panske, Paul, ed. Handfesten der Komturei Schlochau. Danzig: A. W. Kafemann, 1921.

Targowski, Michał. Na prawie polskim i niemieckim. Kształtowanie sie ziemskiej własności szlacheckiej na Pomorzu Gdańskim w XIII-XVI wieku. Warszawa: DiG, 2014.

Thielen, Peter G., ed. Das grosse Zinsbuch des Deutschen Ritterordens (1414-1438). Marburg: N. G. Elwert, 1958.

Ulanowski, Bolesław, ed. Visitationes bonorum archiepiscopatus necnon capituli Gnesnensis saeculi XVI. Kraków: nakł. Polskiej Akademji Umiejętności, 1920.

Wolff, Adam. „Projekt instrukcji wydawniczej dla pisanych źródeł historycznych do połowy XVI wieku”. Studia Źródłoznawcze 1 (1957): 155-184.

Ziesemer, Walther, ed. Das grosse Ämterbuch des Deutschen Ordens. Danzig: A. W. Kafemann, 1921. 